\title{
Los métodos de inferencias en la construcción de un sistema cognitivo artificial determinístico
}

\author{
The methods of inferences in the construction of a deterministic artificial cognitive
}

\author{
system
}

\author{
Sonia I. Mariño \\ Departamento Informática y Computación, Universidad Nacional del Nordeste, Argentina. \\ Correo-e: simarinio@yahoo.com
}

\begin{abstract}
Resumen-El artículo aborda una conexión entre los métodos de inferencia y su representación en el sistema experto determinista como mecanismo de razonamiento de un sistema cognitivo artificial. Se incluye la metodología empleada en su desarrollo. Como producto se detalla la propuesta que enlaza la inducción, la deducción, la abducción y la analogía en la construcción de un sistema experto, presentando una visión integradora $e$ interdisciplinaria de la epistemología, metodología de la investigación y una tecnología simbólica de Inteligencia Artificial. Finalmente, se expresan algunas reflexiones.
\end{abstract}

Palabras clave- ciencias cognitivas, sistemas basados en conocimiento, sistemas expertos, mecanismos de inferencia

\begin{abstract}
This paper deals with a connection between the methods of inference and its representation in deterministic expert system as artificial cognitive system. It includes the methodology used in its development. The product of the research is the proposal that specifically, link induction, deduction, abduction and analogy in the construction of an expert system, presenting an integrative and interdisciplinary view of epistemology, research methodology and symbolic Artificial Intelligence technology. Finally, some reflections are exposed.
\end{abstract}

Key Word Cognitive science, Knowledge based systems, Expert systems, Inference mechanisms

\section{INTRODUCCIÓN}

En la década de 1950 con el avance intelectual de la definición de una máquina abstracta universal capaz de reproducir el funcionamiento propuesto por Alan Turing, disciplinas como la lingüística, la psicología cognitiva, la fisiología del cerebro, la filosofía en varias de sus ramas, la informática y en especial la Inteligencia Artificial (IA) abordadas desde múltiples miradas por científicos y filósofos componen el hexágono cognitivo definido por Gardner (1995).y se reúnen bajo la denominación de Ciencias Cognitivas (CC), como única ciencia interdisciplinaria.

En [20] se menciona que la CC "reconoce plenamente la legitimidad de las investigaciones sobre el conocimiento mismo, en todos sus niveles, más allá de los límites

Fecha de Recepción: 23 de mayo de 2014

Fecha de Aceptación: 30 de Marzo de 2016 tradicionalmente impuestos por la psicología o la epistemología”. Sus estudios se centran en la obtención de la inteligencia o conocimiento, definiendo sus procesos de adquisición naturales y artificiales y el modo en que éstos se constituyen ( [2], [15], [18]).

Autores como [4], [14], entre otros, mencionan que los paradigmas predominantes de la IA son el simbólico y el conexionista o subsimbólico. Otros autores diferencian entre computación dura, hard Computing o tradicional y Computación Suave o Soft Computing.

En numerosos trabajos se utilizan las tecnologías de la IA en la resolución de una diversidad de problemas de distinta complejidad. Entre éstos se mencionan los vinculados con procesos de predicción, clasificación o identificación.

En el presente trabajo las inferencias estudiadas son las que se entienden como comprendidas en un sistema de clasificación o identificación, y su conocimiento es representado y manipulado por un sistema experto (SE) determinístico denominado en el trabajo como Sistema Cognitivo Artificial (SCA). Estos sistemas se corresponden con la definición de sistemas inteligentes simbólicos expuestos por [20].

Cabe recordar los componentes de un SE son la memoria de trabajo, la base de conocimiento, el motor de inferencia, el subsistema de aprendizaje, el subsistema de explicación, el control de coherencia, cuyas funciones son explicitadas en numerosos textos ([4], [14]), y en este trabajo adaptado para el propósito que guía su elaboración. En todo SE se definen: las variables evidenciales y sus valores que representan las características a través de las cuales se aplica el proceso de razonamiento, y la variable objetivo y sus valores que simbolizan las distintas clases o categorías metas comprendidas por el SCA.

Se parte de la premisa que un SCA se corresponde con un modelo de razonamiento no monotónico ([5], [9]). En este trabajo, las leyes del dominio de conocimiento abstraído son representadas por reglas en la base de conocimiento (BC) administradas por el motor de inferencias (MI); y algunas 
pueden ser tentativas, sujetas a excepciones, a anomalías y a cambios.

A continuación se establecen algunas definiciones referentes a un SCA desde la perspectiva de los modos de razonamiento de la lógica ampliativa. Siguiendo a [5], un SE se define como un "modelo de razonamiento de un agente idealmente racional AR, el cual tiene conocimiento lógico 0 (i.e., deductivamente válido) $\mathrm{k}$ y además tiene conocimiento revisable, es decir, conocimiento que expresa opiniones o tendencias generales, pero que pueden tener excepciones"

por ello, se admite que aspectos no contemplados originalmente tratados como singularidades deben ser representados. Es decir, al iniciar la construcción del SCA pueden existir aspectos desconocidos del dominio del conocimiento elegido, y como consecuencia de su evaluación y su aplicación podrán surgir evidencias ante los cuales el SCA deberá ser capaz de invalidar o revisar las conclusiones obtenidas a partir del conocimiento administrado.

Además, un SCA puede definirse como una "teoría no monotónica $\mathrm{T}$ compuesta por un conjunto de reglas derrotables" [8], y pertenecientes al campo del denominado razonamiento revisable, no monotónico, argumentativo, default, entre otras acepciones. Por lo expuesto, las reglas pueden no ser correctas y refutadas al aparecer nuevos casos que implican en su construcción y evaluación inferencias como abducción, inducción, analogía, deducción, y que se suceden en un continuo. Parafraseando a [13] las reglas que componen un SCA pueden ser falsables.

En [17] se sistematiza las propuestas de Peirce al recuperar al corpus de las inferencias lógicas la abducción y de Hegel al proponer el valor de la analogía en la producción de lo nuevo. Esto último en desavenencia con Peirce (1903, 171), quien considera a "la abducción como la única operación lógica que incorpora nuevas ideas".

La abducción actúa como lógica del razonamiento sintético, es un modo de inferencia ampliativa, su resultado añade ideas nuevas al conocimiento existente ([1], [7], [17]). Además, siguiendo a [17] se postula que la analogía también es una inferencia a través de la cual se incorporan novedades en el cuerpo de conocimiento abordado.

En [17] se fundamenta su propuesta al considerar las cuatro formas inferenciales (deducción, inducción, abducción y analogía) no como independientes entre sí sino operando cada una ellas en el proceso de investigación y de construcción de conocimiento. Idea, que resulta superadora del método hipotético-deductivo como método específico de toda producción que pretende alcanzar el lugar de la cientificidad. Por su parte, en [6] se expresa que los patrones de inferencia ampliativa más usuales y de mayor uso en los sistemas inteligentes, cumplen con objetivos diferentes y en ciertos puntos complementarios.

Se amplia la reflexión epistemológica tradicional - que diferencia entre contexto de descubrimiento y validaciónfundamentando la propuesta al considerar a la praxis científica como transformadora del mundo [10]. Este autor [10] distingue cuatro contextos en la actividad tecno-científica a los que denomina: de educación, de innovación (descubrimiento), de evaluación, de aplicación. Considera que el: i) contexto de educación, implica la enseñanza de conceptos lingüísticos e imágenes científicas, técnicas operatorias y manejo de equipos. Además involucra la difusión y la divulgación reflejándose en un número mayor de destinatarios conjuntamente de los involucrados profesionalmente con el tema; ii) contexto de innovación, es decir todo descubrimiento o invento renueva la realidad; iii) contexto de evaluación, dado que la justificación se amplía con la valoración del descubrimiento o invento; iv) contexto de aplicación, la notabilidad de la ciencia aplicada, la técnica y la tecnología ha transformado al mundo actual.

En este trabajo se reflexiona en torno a cómo las inferencias: inducción, deducción, abducción y analogía, se presentan en la construcción de un SCA que implementa como mecanismo de razonamiento un sistema experto determinístico, vinculándolo al contexto de innovación o descubrimiento siguiendo la propuesta de [10].

\section{METODO}

La metodología aplicada en el desarrollo del trabajo, constó de las siguientes fases:

- Revisión sistemática de la bibliografía respecto al abordaje de los métodos de inferencias.

- Indagación exploratoria referente a los modos de producir conocimientos en el proceso de construcción de un SCA que implementa como estrategia de razonamiento un SE determinístico.

- Reflexión, como proceso orientado a elaborar un modelo conceptual que explicite cómo las inferencias se presentan en la construcción de un sistema experto determinístico asociado al contexto de innovación según [10].

\section{RESULTADOS}

La representación y simulación de problemas pertenecientes a cualquier dominio del conocimiento desde la perspectiva de los fundamentos, modelos y métodos de la IA pueden abordarse mediante los procesos de investigación científicotecnológica.

En la Figura 1 se establece una analogía entre las etapas involucradas en un modelo de proceso software de construcción de un SCA y los contextos de innovación, evaluación y aplicación siguiendo a [10]. En este apartado se 
reflexiona se aborda el contexto de innovación desde la perspectiva de [10] y vinculándolo con las inferencias producidas en su generación.

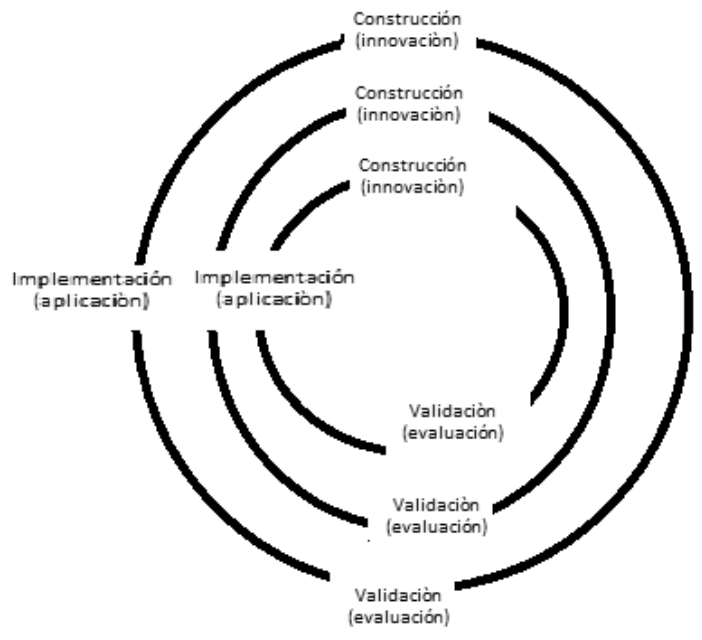

Fig. 1 Propuesta de vinculación de un modelo evolutivo de proceso software de construcción de un SCA y los contextos de innovación, evaluación y aplicación siguiendo a [10]

(Fuente: elaboración propia).

El proceso de construcción del SCA, es decir, su análisis, diseño y desarrollo, desde la Ingeniería del Software y la Inteligencia Artificial, se vincula al contexto de innovación, dado que produce una renovación en el modo de representar y manipular el conocimiento de un dominio delimitado, y en el cual intervienen para su conceptualización sujetos influenciados por un determinado entorno socio-económicocultural. El contexto de evaluación implica la validación y verificación desde la perspectiva computacional y desde la representación y procesamiento del conocimiento del dominio elegido para la simulación. Finalmente, la implementación del SCA se correspondería con el contexto de aplicación.

En este trabajo, se establece que quienes intervienen en la construcción de un SCA se identifican como especialistas o Expertos en el Dominio del Conocimiento (EDC), Ingeniero del Conocimiento (IC), Ingeniero en Sistemas (ISist) representados por distintos especialistas de la disciplina como diseñadores, desarrolladores, expertos en seguridad entre otros perfiles, y los usuarios finales (UF) destinatarios del artefacto inteligente para mediar el proceso de identificación.

El IC aplica técnicas de la Ingeniería del Conocimiento a fin de obtener los conocimientos tácitos de los EDC y explicitarlos, en conjunto con el ISist, en un artefacto inteligente. Es decir, formalizarlo y representarlo aplicando algún esquema, como las reglas que conforman la $\mathrm{BC}$ y sus vinculaciones plasmadas en el MI del SCA determinístico.

Es difícil precisar aquella inferencia que acontece en primer término en todo razonamiento humano. Se propone a la analogía, dado que a través de metáforas se análoga con situaciones reales y similares (casos en estudio) que permiten derivar las reglas que componen la BC. Siguiendo a [17] si la analogía hace posible el descubrimiento o ideación de nuevas Reglas (o hipótesis); esta debe considerarse como el primer momento inferencial en la generación de un SCA.

Las nociones expuestas, permitirían proponer nuevas reglas que crean o modifican la BC (según el ciclo del espiral en un modelo de proceso software que se aborde). En este sentido, existe un objeto semejante (el caso) a uno conocido e identificado como perteneciente a un categoría o clase y dado que a través del mecanismo implementado en el MI no se verifican las reglas establecidas para su determinación (clasificación o identificación) como perteneciente a una categoría o valor de la variable objetivo, da lugar a proponer una nueva idea que se plasma en una(s) regla(s) y su correspondiente tratamiento por el MI.

La abducción es la inferencia capaz de conectar el mundo empírico de las observaciones, en decir aquellas realizadas sobre los casos en estudio, con las configuraciones o totalidades relacionales expresadas como reglas de una BC. Hace funcionar una Regla (o hipótesis) disponible conjeturando que unos rasgos anómalos son comprensibles como Caso de una Regla dada ([17], [19]). Además, siguiendo a [1] la abducción es la inferencia que permite idear nuevos casos (expresados en reglas) o generar ruidos, implicando cambios en la representación y administración del conocimiento explicitado en el SCA. Es el modo de inferencia que se presenta - antes que la deducción y la inducción- en la construcción de un SCA.

Como se expresó previamente [12], aun cuando el punto lógico de la abducción es una falacia de la afirmación del consecuente, es de todos modos un instrumento de búsqueda de conocimiento que se funda en verdades científicas y en la praxis del sujeto, en este caso derivada de las observaciones realizadas sobre los casos contemplados para la construcción del SCA. Autores como [3], [9], [17] mencionan que el mecanismo disparador de la abducción pueden ser el hecho novedoso o sorprendente, o la anomalía.

Cada una de las aseveraciones mediante las cuales se establece la presencia o ausencia de una característica de una variable o el valor que éste asume, se desprende de la sistematización -y consecuentemente de la explicitación- de las observaciones realizadas sobre los casos o conocimiento acordado por los EDC y utilizado para construir la BC.

La inducción tiene por objetivo encontrar una regla general o patrón a partir de un conjunto de casos particulares, ejemplos o instancias. El razonamiento inductivo genera un conjunto de reglas a priori a partir de la observación de un conjunto de casos particulares precisándose cierta regularidad o patrón. Es decir, el conocimiento elicitado origina la formulación de expresiones generales, representadas en las reglas del SCA. Se 
estaría ante la presencia de una inferencia inductiva, que es la ley de presunta validez universal obtenida de la observación de uno o más casos y de la cual no se puede asegurar que la conclusión sea verdadera en general.

El método inductivo, explica los hechos observados. Se fundamenta en la reproductibilidad mediante la comunicación y publicación de los hallazgos y la falsabilidad, es decir, si se obtienen resultados diferentes a los predichos, la hipótesis debe ser negada. Su aplicación es válida siempre que se carezca de algún caso que no cumpla el modelo propuesto, por ello la disponibilidad y evaluación de otros casos daría lugar a revisar y modificar la BC y el MI. En palabras de [17] aun realizada una

"veraz descripción de los eventos particulares que sostienen nuestras premisas, la conclusión general que pretendemos derivar podría resultar falsa, dado que nos falta información sobre los restantes elementos del conjunto"

que podrían ser desconocidos, y además, “....si el conjunto es infinito, por más que agreguemos más observaciones a nuestra muestra, no por eso habremos aumentado la probabilidad de que sea verdadera".

Por otra parte, la inducción implica la abducción de un caso y deducción de un rasgo. Lo expuesto, refleja la necesidad de concebir SCA no monotónico. Además, para asegurar la validez de una regla o cadena de reglas que componen la BC, se requieren de numerosas observaciones, disponer de más datos de la realidad se logrará al aumentar la disponibilidad y diversidad de los casos, destacándose la experiencia de los sujetos cognoscentes especialistas.

Cabe aclarar, en el proceso de construcción de la BC del SCA el especialista, explicita su experticia, pensamientos y conocimientos que implican razonamientos y pueden no ser observables a simple vista. Sin embargo pueden reconstruirse a partir de las acciones o explicitaciones realizadas. En numerosas ocasiones el recurso humano en formación u otros sujetos intervinientes en la construcción, evaluación y uso de un SCA pueden no detectar estos procesos cognitivos implicados.

Si una Base de Conocimiento (BC) es un conjunto de reglas y siguiendo a [19],

"la Regla contiene una afirmación sobre un sujeto general (es decir, sobre un conjunto), entonces, vale describir a la inferencia deductiva, como una inferencia de particularización: que va de un saber en general, a su aplicación en particular".

Es decir, un SCA como todo se constituiría en una ley general o teoría que incorpora el conocimiento de sujetos especialistas del dominio elegido y éste es explicitado mediante su representación en su BC y MI. Cabe aclarar que este SCA es un sistema inteligente rebatible, es decir, pueden surgir casos que derroten el conocimiento representado en él.
En referencia a la deducción, ésta es analítica; la información de la conclusión está sugerida en las premisas. Es un modo de inferencia predictiva o explicativa, según se haya realizado antes o después de la comprobación de los hechos deducidos [11]. Es también considerada por Peirce, como un modo de inferencia ampliativa aunque no puramente sintética.

En resumen, se parte de la propuesta que la construcción de un SCA determinístico es un proceso de explicitación de conocimientos creados por la mente de los sujetos cognoscentes intervinientes. Se podría proponer a la analogía como forma de inferencia que formaliza las ideas, experiencias acordadas por los EDC, y se constituye en el primer paso de este proceso de construcción de conocimiento que surge en la praxis, en la investigación empírica. A continuación se transitaría hacia una inferencia abductiva, que permitiría generar hipótesis plausibles expresadas en reglas y otras derivadas de procesos de observación inductivos, obteniéndose un instrumento de aplicación en el dominio de conocimiento elegido, el SCA, sobre el cual se pueden aplicar otros procesos inferenciales.

Finalizada la construcción de un SCA determinístico, éste como totalidad, representa un sistema de razonamiento deductivo dado que el conocimiento se encuentra representado en sus reglas que encarnan generalidades y se corresponde con un modelo de la lógica ampliada. Además, dispone de un componente que facilita la construcción de nuevo conocimiento -el subsistema de adquisición de conocimientos-, como resultado de la puesta en escena de otros razonamientos como son la analogía, la abducción o la inducción.

\section{CONCLUSIONES}

En el presente trabajo se expuso una aproximación desde una perspectiva conceptual ilustrando la puesta en escena de las inferencias asociadas a los distintos momentos de un modelo de proceso de construcción de un sistema experto como dispositivo cognitivo artificial determinístico. Siguiendo a [10] se corresponde al contexto de innovación o descubrimiento, simulándose conceptualmente los distintos modos de inferencia, que ni se presentan definidos ni delimitados sino se suceden en un continuo espiral de conocimiento.

El SCA determinístico, como totalidad, puede conceptualizarse como un sistema deductivo rebatible no monotónico, las reglas que lo componen representan generalidades creadas a partir del conocimiento en un determinado tiempo y espacio, atendiendo a la muestra disponible y no al universo del dominio objeto de estudio, dado que algunos casos que lo componen pueden desconocerse al momento de su diseño, desarrollo, evaluación o utilización. Por lo expuesto, permitiría apoyar la toma de decisiones en los procesos de clasificación o identificación 
contemplados por el artefacto inteligente $\mathrm{y}$, aceptando que puede modificarse ante la presencia de nuevos casos, plasmándose en su revisión y adecuación para representar la nueva realidad abstraída, requiriéndose nuevos ciclos de una espiral de conocimiento.

Cabe destacar que un SCA es producto del intelecto humano, de la creatividad de los expertos del dominio del conocimiento, del Ingeniero del Conocimiento, de los Ingenieros de Sistemas. Formaliza y representa el capital intelectual plasmándose en una herramienta computacional, por lo que podría pensarse como un modelo del entendimiento en un determinado tiempo y espacio.

Dado que en este trabajo, se reflexionó respecto a la construcción del SCA en referencia a los distintos modos de razonamiento que se suceden y complementan en el contexto de innovación o descubrimiento, a partir de lo expuesto se continuará con

- la determinación de las inferencias involucradas en el contexto de evaluación.

- la determinación de las inferencias involucradas en el contexto de aplicación.

- la validación empírica, que demostrará como el SE, tecnología inteligente incorporada en el SCA, posibilita una herramienta complementaria para simular y apoyar la toma de decisiones de los expertos en el dominio del conocimiento, recursos humanos en formación e interesados en la temática.

Es así como desde una mirada trandisciplinaria, integrando la Epistemología, la Metodología de la Investigación y la Inteligencia Artificial se espera aportar un modelo holístico y su objetivación en un sistema inteligente para abstraer y simular complejos problemas del mundo real.

\section{REFERENCIAS}

[1] A. Aliseda-Llera, "La abducción como cambio epistémico: C. S. Peirce y las teorías epistémicas en Inteligencia Artificial". Analogía 12 (1998), 125144, 1998.

[2] A. Aliseda-Llera, "Emerge una nueva disciplina las Ciencias Cognitivas". Ciencias octubre diciembre 88: 22-31, 2007.

[3] A Bar, "Abducción. La Inferencia del Descubrimiento". Cinta de Moebio, 12:169 - 174, 2001.

[4] E. Castillo, J. M. Gutiérrez y A. S. Hadi, Sistemas expertos y modelos de redes de probabilísticas. Academia de Ingeniería, 1996.

[5] C. Delrieux, "Formalización del Razonamiento Hipotético e Inductivo en Teoría de la Ciencia". I Congreso Argentino de Ciencias de la Computación, 1995.
[6] C. Delrieux, "Una interpretación plausibilística de los patrones ampliativos de Inferencia". Congreso Argentino de Ciencias de la Computación, 1997.

[7] C. Delrieux, "La relevancia de la lógica relevante en el razonamiento no monotónico". III Workshop de Investigadores en Ciencias de la Computación, 2001.

[8] C. Delrieux, "Razonamiento abductivo en teorías no monotónicas". IX Congreso Argentino de Ciencias de la Computación, p. 1628-1639, 2003.

[9] C. Delrieux, "Inducción y analogía en el razonamiento revisable". VII Workshop de Investigadores en Ciencias de la Computación, p. 373-377, 2005.

[10] J. Echeverría, J. Filosofía de la ciencia, Ed. Akal, Madrid, 1995.

[11] C. G. Hempel, y P. Oppenheim, "The Logic of Explanation". Philosophy of Science, 15: 135-175, 1948.

[12] Autor1.

[13] K. Popper, K. La lógica de la investigación científica, 1935.

[14] S. Russell y P. Norving, Inteligencia Artificial: Un Enfoque Moderno, Ed. Prentice Hall Hispanoamericana S.A., 2004

[15] Sáenz, T W. (2005). Las ciencias y tecnologías modernas. Su convergencia. pp. 7-26 en Rodolfo Faloh Bejerano (coord.). Gestión de la innovación. Una visión actualizada para el contexto Iberoamericano. Ed. Academia,

[16] J. Samaja, "El papel de la hipótesis y de las formas de inferencia en el trabajo científico", [Online], Disponible: http://publicaciones.fba.unlp.edu.ar/wpcontent/uploads/2011/11/El-papel-de-lahip\%C3\%B3tesis-y-de-las-formas-de-inferencia.pdf (consulta: 2013, Mayo 16)

[17] J. Samaja, Epistemología y Metodología de la Investigación Científica Ed. EUDEBA. 2003

[18] J. Samaja, Materiales del Doctorado en Ciencias Cognitivas. Facultad de Humanidades. Universidad Nacional del Nordeste. Inédito, 2006.

[19] R. C Ynoub, “Abducción y falsacionismo: aportes de la teoría de la abducción de Peirce para iluminar los límites del falsacionismo popperiano". III Jornadas "Peirce en Argentina", 2008.

[20] F. J. Varela F. J, Conocer: las ciencias cognitivas, tendencias y perspectivas, 1990. Barcelona, Ed. Gedisa. 